\title{
Empfehlungen für Schwimmen als Bewegungstherapie bei kardialen Erkrankungen
}

\author{
Eine Therapieoption, auch für übergewichtige Personen
}

Schwimmen ist für Herzkreislaufpatienten eine weit verbreitete Bewegungstherapie, die insbesondere von übergewichtigen Personen sehr gern angenommen wird, da der Großteil des Übergewichts durch das Wasser getragen wird.

Die Voraussetzungen für das Schwimmen von Herz-Kreislauf-Patienten sind folgende:

\section{Schwimmtechnik}

Die Patienten sollten schwimmen können, d.h. es erscheint unsinnig Nichtschwimmern mit Herz-Kreislauferkrankungen z.B. nach einem kardialen Ereignis, wie einem Herzinfarkt, das Schwimmen primär beibringen zu wollen, obwohl dies mehrmals versucht wurde, da dies für die Patienten auch im Wasser einen inadäquat großen Stress nach sich zieht, verbunden mit hohen Katecholaminspiegeln und konsekutiv der potenziellen Möglichkeit von schwerwiegenden Herzrhythmusstörungen. Das bedeutet mindestens Brustschwimmen sollte von diesen Patienten sicher gekonnt werden!

\section{Rhytmusstörungen}

Ein weiterer, eher theoretischer Ansatz ist, dass Patienten, die in der Anamnese schwerwiegende Rhythmusstörungen aufweisen, wie z.B. Kammertachykardien, nicht schwimmen gehen sollten. Hier wird spekuliert, dass mit dem Eintauchen in das Wasser, z.B. durch das Herabsteigen über Stufen oder über eine Leiter, durch den von unten nach oben zunehmenden hydrostatischen Druck das Blut aus den Beinen ausgepresst wird und ein vermehrtes, rechtsatriales venöses Pooling entsteht mit einem Überangebot an Blut, welches konsekutiv zu einer vermehrten Dehnung des rechten Vorhofes beziehungsweise der rechten Herzkammer zur Bewältigung dieses erhöhten Blutrückflusses führen kann, ein Umstand, der ebenfalls Rhythmusstörungen induzieren soll. In der Praxis konnten wir dies aber weder durch schwimmtelemetrische Beobachtungen, noch durch akute Zwischenfälle beobachten, sodass diese Überlegung eher theoretischer Natur ist.

\section{Leistungsfähigkeit}

Eine weitere, insbesondere in den diversen Lehrbüchern immer wieder erwähnte Forderung, ist eine Leistungsfähigkeit am Fahrradergometer von 100 Watt oder mehr. Auch hier sollte eher individuell nach Körpergewicht und Leistungsfähigkeit vorgegangen werden. Mit anderen Worten: bei uns dürfen auch Patienten mit 75 Watt schwimmen, wenn sie leichtgewichtig und nicht herzinsuffizient sind.

\section{Umgebungsbedingungen}

Als letzter, aber wichtiger Punkt sind die Umgebungsbedingungen für das
Schwimmen zu erwähnen: Das Wasser sollte keineswegs zu kalt sein, da im kalten Wasser bei entsprechenden Patienten eine Kälteangina induziert werden kann beziehungsweise inadäquate RRAnstiege beobachtbar sind, optimale Wassertemperaturen sind zwischen 26 und 30 Grad Celsius.

Weiters sollte das gesamte Schwimmbad eine maximale Tiefe von 1,40 Meter nicht überschreiten, damit gewährleistet ist, dass ängstliche Patienten überall stehen können beziehungsweise sollte es zu schwerwiegenden Rhythmusstörungen oder Unwohlsein kommen, diese sich noch bemerkbar machen können und nicht lautlos untergehen und ertrinken. Dementsprechend gibt es z.B. in allen österreichischen Herz-Kreislauf-Rehabilitationszentren auch kein Schwimmen ohne Badeaufsicht, Notruftaste und Notfallinstrumentarium in Reichweite. Schlussendlich ist festzuhalten, dass es im RZ-Austria in Bad Schallerbach in den letzten 20 Jahren beim Schwimmen $\mathrm{zu}$ keinem einzigen tödlichen Zwischenfall gekommen ist, was zweifellos auch auf die obengenannten Richtlinien zurückgeführt werden kann.

Korrespondenz:

Prim. Univ.Prof. Dr. Schmid Peter

Rehabilitations- und Kurzentrum Austria - Ärztliche Leitung Versicherungsanstalt öffentlich Bediensteter Stifterstraße 11 4701 Bad Schallerbach

Telefon: (07249) $42541-84100$

Fax: (07249) 425 41-84888

E-Mail: peter.schmid@bva.sozvers.at 